\title{
The association of chiral characteristic with drug withdrawal due to safety: a comparative analysis
}

\author{
Ayfer Bahar ${ }^{1}$, Volkan Aydin ${ }^{2}$, Caner Vizdiklar ${ }^{1}$, and Ahmet Akici ${ }^{1}$ \\ ${ }^{1}$ Marmara University School of Medicine \\ ${ }^{2}$ Istanbul Medipol University
}

February 11, 2022

\begin{abstract}
Aim: Chirality of drugs might be associated with safety issues through pharmacokinetic or pharmacodynamic variations, interactions, or direct toxicological responses. This study aimed to examine chiral status of the drugs withdrawn from the market. Methods: We searched the literature regarding withdrawn drugs between 1950-2020 due to safety-related issues and identified 395 drugs. We examined their chirality and assigned into one of three categories: achiral compound, chiral mixture, and pure enantiomer. We compared their distribution at ATC-1 level, duration on the market, and adverse drug reactions leading to their withdrawal. Results: We identified that $52.4 \%(\mathrm{n}=207)$ of withdrawn drugs were achiral, whereas $27.6 \%(\mathrm{n}=109)$ were chiral mixtures and $20.0 \%(n=79)$ were pure enantiomers. The mean duration on the market was $24.6 \pm 27.5$ years. The groups did not differ in terms of mean duration on the market. Chiral mixtures were significantly more withdrawn than were achirals in cardiovascular system drugs $(17.4 \%$ vs. $7.7 \%, \mathrm{p}=0.01)$. In musculoskeletal system drugs, pure enantiomers were significantly less withdrawn $(2.5 \%)$ compared to achirals $(12.6 \%, \mathrm{p}=0.01)$ and chiral mixtures $(11.9 \%, \mathrm{p}=0.03)$. Hepatotoxicity was significantly less common in pure enantiomers $(5.4 \%)$ compared to chiral mixtures $(12.7 \%, \mathrm{p}=0.04)$ and achirals $(17.0 \%$, $\mathrm{p}<0.01)$. Cardiovascular toxicity was significantly more common in chiral mixtures $(14.5 \%)$ compared to that in achiral drugs $(7.5 \%, \mathrm{p}=0.02)$. Conclusion: Our study showed slightly higher representation of chiral mixtures among withdrawn drugs over pure enantiomers. The assessment of withdrawal reasons further indicates higher tendency of chiral mixtures towards hepatotoxicity and cardiovascular toxicity.
\end{abstract}

The association of chiral characteristic with drug withdrawal due to safety: a comparative analysis

Running title: Chiral status of withdrawn drugs

Ayfer Bahar ${ }^{*}$, Volkan Aydin² ${ }^{*}$, Caner Vizdiklar ${ }^{1}$, Ahmet Akici $^{1}$

Department of Medical Pharmacology, School of Medicine, Marmara University, Istanbul, Turkey

Department of Medical Pharmacology, International School of Medicine, Istanbul Medipol University, Istanbul, Turkey

* The authors equally contributed to this paper.

Correspondence: Prof Ahmet Akici, MD

Department of Medical Pharmacology, Marmara University School of Medicine, Maltepe 34854, Istanbul, Turkey.

Phone: +902165557700; e-mail:aakici@marmara.edu.tr 
Ethical approval: This study was approved by Ethics Committee for Non-interventional Clinical Studies of Marmara University Institute of Health Sciences (Approval number: 16.11.2020-91).

Acknowledgements : We thank to Dr. Igho Onakpoya and his co-workers for their tremendous efforts and earlier studies on withdrawn drugs that inspired us to investigate another aspect of the subject.

Competing Interests: The authors declare they have no competing or conflict of interest.

Funding: This research did not receive any specific grant from funding agencies in the public, commercial, or not-for-profit sectors.

Contributions: Conception and design: AB, AA, VA; Acquisition of data: AB, CV, VA; Analysis and interpretation of data: VA, AB, CV; Writing first draft: VA, AB, CV, AA; Critical review and supervision: AA, VA; Final approval to the submitted version of the manuscript: AB, VA, CV, AA.

Data availability: The data that support the findings of this study are available from the corresponding author upon reasonable request.

Keywords: pharmacovigilance, pharmacoepidemiology, drug regulation, stereoisomerism

\section{Word count: 2682, Table count: 4, Figure count: 1}

\section{What is known about this subject}

Chiral status of drugs is postulated to be among factors that influence drug safety through pharmacokinetic/pharmacodynamic changes, toxicities or drug interactions.

There has not been any systematic analysis that investigated safety issues/benefits attributed to chiral status.

\section{What this study adds}

Chiral distribution of the withdrawn drugs could differ by the reasons for withdrawal, such as increased tendency of cardiovascular ADRs and hepatotoxicity in chiral mixtures.

Further investigation of potential relationships between chirality of drugs and ADR mechanisms might eventually lead to improvements of safety standards in new drug development processes.

\section{ABSTRACT}

Aim: Chirality of drugs might be associated with safety issues through pharmacokinetic or pharmacodynamic variations, interactions, or direct toxicological responses. This study aimed to examine chiral status of the drugs withdrawn from the market.

Methods: We searched the literature regarding withdrawn drugs between 1950-2020 due to safety-related issues and identified 395 drugs. We examined their chirality and assigned into one of three categories: achiral compound, chiral mixture, and pure enantiomer. We compared their distribution at ATC-1 level, duration on the market, and adverse drug reactions leading to their withdrawal.

Results: We identified that $52.4 \%(\mathrm{n}=207)$ of withdrawn drugs were achiral, whereas $27.6 \%(\mathrm{n}=109)$ were chiral mixtures and $20.0 \%(\mathrm{n}=79)$ were pure enantiomers. The mean duration on the market was $24.6 \pm 27.5$ years. The groups did not differ in terms of mean duration on the market. Chiral mixtures were significantly more withdrawn than were achirals in cardiovascular system drugs ( $17.5 \%$ vs. $7.7 \%, \mathrm{p}=0.01)$. In musculoskeletal system drugs, pure enantiomers were significantly less withdrawn (2.5\%) compared to achirals (12.6\%, $\mathrm{p}=0.01)$ and chiral mixtures $(11.9 \%, \mathrm{p}=0.03)$. Hepatotoxicity was significantly less common in pure enantiomers (5.4\%) compared to chiral mixtures $(12.7 \%, \mathrm{p}=0.04)$ and achirals $(17.0 \%, \mathrm{p}<0.01)$. Cardiovascular toxicity was significantly more common in chiral mixtures (14.5\%) compared to that in achiral drugs $(7.5 \%$, $\mathrm{p}=0.02)$.

Conclusion: Our study showed slightly higher representation of chiral mixtures among withdrawn drugs over pure enantiomers. The assessment of withdrawal reasons further indicates higher tendency of chiral 
mixtures towards hepatotoxicity and cardiovascular toxicity.

\section{INTRODUCTION}

Chirality is an important geometric characteristic of the objects within biological systems including amino acids, carbohydrates, and lipids as well as drugs. In the latter, chirality might be associated with safety issues through pharmacokinetic or pharmacodynamic variations, drug interactions, or direct toxicological responses . Chiral drugs consist of racemic mixtures, non-racemic mixtures, or pure enantiomers . Unlike achiral drugs with no chiral center, this stereoisomeric chemistry allows the opportunity to manipulate their composition or molecular chirality to enhance efficacy and/or overcome tolerability problems . A typical example of improving clinical efficacy could be given as selective H1-receptor antagonist, cetirizine, whose R-enantiomer levocetirizine has 30-fold higher binding affinity and lower renal clearance compared to its parent racemic mixture. On the other hand, thalidomide represents a well-known dramatic example of druginduced toxicity, with R-enantiomer responsible for the intended sedative effect and S-enantiomer for the tragic phocomelia .

Use of pure enantiomers offers advantages including dose reduction, simplification of dose-response relationship, diminution of interindividual variability and toxicity from inactive enantiomers . In fact, regulatory drug authorities encourage such chemical designations for novel drug development . In addition, some racemic mixtures were undergone chiral switch, where their pure enantiomers were launched with same/similar indication. These have led to increased share of pure enantiomers worldwide though still many racemic and non-racemic mixtures are present. While several clinical efficacy and/or safety benefits have been attributed to pure enantiomer drugs, there has been no systematic analysis that investigated these aspects with respect to chirality. In this study, we aimed to examine chiral status of the drugs withdrawn from the market.

\section{METHODS}

In this pharmacoepidemiologic study, we collected and analysed retrospective descriptive drug safety data. Prior to data collection, ethical approval was obtained from Marmara University Institute of Health Sciences, Non-Interventional Clinical Studies Ethical Committee (approval number: 16.11.2020-91).

We examined chemical structures regarding chirality status of the drugs, which were withdrawn from market due to adverse effects. Drugs withdrawn between 1950 and 2020 in the world due to safety-related issues were identified via literature search. Those which were withdrawn between 1950 and 2014 were obtained from a systematic review conducted by Onakpoya et al., including a total of 462 drugs/products . Drugs/medicinal products that contain inorganic compounds, proteins, vaccines, polymers, human tissue extracts, herbal and cell-based preparations as active substances, and combinations of two active substances that show different chiral status from each other were excluded from the study. Remaining 389 drugs, i.e., the ones that comprise single active substance $(\mathrm{n}=383)$ and the combinations of active substances showing similar chiral characteristics $(\mathrm{n}=6)$, were included. Using a methodology similar to the aforementioned review, literature search was conducted for drugs withdrawn between 2015 and 2020, which resulted in six drugs meeting those criteria (Supplementary Table 1). Thus, a total of 395 drugs/medicinal products were included in the first part of the study. Chiral characteristics and distribution of those drugs at ATC-1 level, as well as the distribution of their duration on the market, and adverse drug reactions (ADR) leading to their withdrawal were examined.

Chirality status of the drugs were identified via information on the "Inxight: Drugs" database of U.S. National Institute of Health . For the ones that are not available on that database, chirality status was determined after literature search. The mean time to withdrawal (TtW) and the duration (period from introduction to the calendar year of 2020) and year of launch of drugs according to chirality status were examined. According to chiral characteristics, the drugs were divided into three main groups as "achiral", "chiral mixture" and "pure enantiomer". Also, TtWs of drugs were evaluated categorically in six distinct time periods as "0-20/21-40/4160/61-80/80-100/[?]100 years".

Chiral distribution of the withdrawn drugs was evaluated and compared at ATC-1 level. Before that, we 
re-classified drugs with multiple ATC codes or without any code by adding them to the most appropriate ATC class according to the indication led to withdrawal, whenever possible. Those drugs were evaluated and compared first as "achiral" and "chiral", and chiral ones were further separated into two to form three main groups as "achiral drugs", "chiral mixtures" and "pure enantiomers" to analyse in detail.

ADRs leading to withdrawal of drugs were evaluated in 20 subgroups (i.e., cardiovascular, genitourinary, gastrointestinal, respiratory, neurological, haematological, dermatological, psychiatric, endocrine, ophthalmic, musculoskeletal, and other ADRs, along with hepatotoxicity, nephrotoxicity, genotoxicity, drug abuse, carcinogenicity, and death). These were distributed and compared by the chirality status (achiral/chiral mixture/pure enantiomer) of the related drugs.

\section{Statistical analysis}

All data were analysed using IBM SPSS Statistics for Windows, Version 22.0 (IBM Corp., Armonk, NY, USA) and GraphPad Prism version 5.0 for Windows (GraphPad Software, San Diego, CA, USA) software. Analysed data were expressed as numbers and percentages or mean + - standard deviation values, where appropriate. Frequency analysis was used for statistical evaluation and categorical variables were compared using chi-square test. For continuous variables, normality of distribution was evaluated by D'AgostinoPearson and Shapiro-Wilk analyses. Normally distributed data were compared using one-way analysis of variance (ANOVA) test with Tukey's post-hoc test, whereas Kruskal-Wallis test was used with Dunn's posthoc test if normal distribution is not applicable. Statistical significance was inferred by an overall $5 \%$ of type-I error level.

\section{RESULTS}

\section{Chirality Status of Withdrawn Drug Groups}

Of the 395 drugs withdrawn, $52.4 \%(\mathrm{n}=207)$ were achiral, $27.6 \%(\mathrm{n}=109)$ were chiral mixtures, and $20.0 \%$ $(\mathrm{n}=79)$ were pure enantiomers. The mean $\mathrm{TtW}$ of all those drugs evaluated for safety were $24.6+-27.5$ (0-226) years, and the time after launch was 57.3+-30.1 (8-267) years.

"N-nervous system drugs" had the highest share (26.3\%) when distributed at ATC-1 level. Five of 13 ATC-1 classes were predominantly chiral. Furthermore, the chiral predominance in "C-cardiovascular system drugs" were statistically significant when compared to other classes $(\mathrm{p}=0.02)$, (Figure 1$)$.

Chiral mixtures were significantly higher in number than achirals in cardiovascular system drugs (17.5\% vs. $7.7 \%, \mathrm{p}=0.01)$. In musculoskeletal system drugs, pure enantiomers were significantly less withdrawn $(2.5 \%)$ compared to achirals $(12.6 \%)$ and chiral mixtures $(11.9 \%),(\mathrm{p}=0.01$ and $\mathrm{p}=0.03$, respectively). On the contrary, pure enantiomers were subject to withdrawal at a higher rate $(8.9 \%)$ compared to chiral mixtures $(1.8 \%)$ in the genitourinary system drugs $(\mathrm{p}=0.04)$, (Table 1$)$.

\section{Chirality Status by Adverse Drug Reactions}

A total of 583 ADRs were among the reasons of withdrawal of the drugs evaluated. Fifty-two and a half percent $(n=306)$ of those were related to achiral drugs, $28.3 \%(n=165)$ to chiral mixtures and $19.2 \%(n=112)$ to pure enantiomers. Among the most encountered ADRs, hepatotoxicity was significantly less common in pure enantiomers $(5.4 \%)$ compared to chiral mixtures $(12.7 \%, \mathrm{p}=0.04)$ and achirals $(17.0 \%, \mathrm{p}<0.01)$. Cardiovascular ADRs were significantly more common in chiral mixtures (14.5\%) compared to that in achiral drugs $(7.5 \%, \mathrm{p}=0.02)$, with no difference from pure enantiomers $(11.6 \%, \mathrm{p}>0.05)$. Drug abuse for a reason of withdrawal was found to be less common in achiral drugs $(5.2 \%)$ than that in chiral mixtures $(13.3 \%, \mathrm{p}<0.01)$ and pure enantiomers $(11.6 \%, \mathrm{p}=0.03)$. In addition, dermatological ADRs were significantly less common in pure enantiomers compared to that in achiral drugs $(1.8 \%$ vs. $8.8 \%, \mathrm{p}<0.01)$ for pure enantiomers (Table 2).

\section{Chirality Status by Temporal Patterns}

The drugs withdrawn were mostly launched between years of 1960-1980 (36.7\%) for all chiral groups. Achiral 
drugs had the highest share in all time periods (48.3\%-70.8\%). Chiral mixtures were more commonly withdrawn from the market than pure enantiomers in all time periods, except those launched before the year 1920 (4.2\% vs. 25.0$)$ and after the year 2020 (5.6\% vs. 33.3\%), (Table 3 ).

Mean TtWs of achiral drugs, chiral mixtures and pure enantiomers were similar $(25.9+-29.1,20.0+-16.9$, and $27.9+-33.8$ years respectively, $\mathrm{p}>0.05$ ). The drugs withdrawn due to abuse had the highest mean TtW (35.1+-28.1 years) and those leading to death had the lowest (11.4+-9.6 years). Achiral drugs withdrawn due to dermatological ADRs had longer mean TtW (28.4+-20.4 years) compared to that in chiral mixtures $(9.3+-10.9$ years, $\mathrm{p}=0.01)$, (Table 4$)$.

\section{DISCUSSION}

We examined chirality of the drugs withdrawn from the market over the last 70 years to uncover the potential reflections of such characteristic on major safety issues that outweigh its intended benefits. We observed that chiral status of withdrawn drug groups at ATC-1 level seem to exhibit subtle differences. On the other hand, assessment of ADRs by chiral category pointed out the tendency of racemic/non-racemic chiral mixtures towards hepatotoxicity and cardiovascular ADRs.

Drugs with a chiral characteristic, either in the form of racemic/non-racemic mixtures or pure enantiomers, constitute more than half $(58 \%)$ of the drugs used. This figure appeared to show modest increments in favour of chiral drugs, as the annual new worldwide approval rates of chiral drugs were reported to range between $50 \%$ to $76 \%$. In our study, $47.6 \%$ of the withdrawn drugs were of the chiral type, which seems to imply that the withdrawal risk of chiral drugs may be slightly less. Pure enantiomer drugs may offer advantage of less complex pharmacological profile with a greater therapeutic index. Nevertheless, our findings showed a comparable ratio of withdrawn chiral mixtures (58\%) and pure enantiomers (42\%), questioning the relative potential advantage of the latter on withdrawal. In fact, the predominant composition among chiral drugs is racemic mixtures. While scarce data was present in the literature, a recent nationwide study from Tanzania reported that $74 \%$ of all chiral drugs available on the market between 2003-2018 were racemates . On the other hand, the upward trend of chiral drugs appears to be mostly driven by up to 15-fold higher introduction of pure enantiomers over racemic mixtures between 2000-2008. Therefore, the inverse association of chiral drugs to withdrawal might be partly attributed to the relatively higher share of pure enantiomers among newly approved drugs. This was further supported by our finding that those launched and withdrawn in the recent 20 years revealed a comparably lesser predominance of pure enantiomers $(33.3 \%)$ over chiral mixtures $(5.6 \%)$.

Chiral distribution of the withdrawn drug groups suggest that the overall pattern was preserved across many of the groups at ATC-1 level with several exceptions. One of these groups belonged to musculoskeletal system drugs, where the rate of withdrawal was higher for chiral mixtures (11.9\%) than that in pure enantiomers $(2.5 \%)$. Musculoskeletal system drugs include a substantial part of non-steroidal anti-inflammatory drugs (NSAIDs), whose majority is formed by racemates. In fact, NSAIDs have been well-recognized to be associated with important safety issues that may result in withdrawal. A study on withdrawn drugs between 1960-1999 reported that 13\% of such drugs were NSAIDs . In fact, NSAIDs were among the most commonly utilized drug groups. This could suggest that putatively lower safety margin of chiral mixtures compared to pure enantiomer drugs may be associated with the emergence of important safety issues in frequently used medications. This might have been contributed by possible over-the-counter or non-prescription use of these drugs as self-medication since we did not identify such difference in cardiovascular drugs, commonly used prescription medicines. The top three best-selling drugs in the United States in 2009 were reported to belong to cardiovascular category and all were pure enantiomers. In addition, chirality-related pharmacodynamic and pharmacokinetic characteristics of cardiovascular drugs were also extensively reviewed in the literature . Nevertheless, our findings suggest that a potential relationship of chirality to withdrawal did not seem likely for cardiovascular mixed and pure enantiomers.

ADRs have a very wide range of potential causes, including chemical structure of the drugs, patient-related factors, concomitantly used drugs or medicinal products, etc. . Possible underlying causes trigger the 
incidence of drug-related problems of directly related tissues and organs. Hepatotoxicity, the most common type of ADR in our study, was the reason for withdrawal near 2.5-fold less likely in pure enantiomers (5.4\%) than that in chiral mixtures (12.7\%). As the major organ for drug biotransformation, the liver comprises majority of drug metabolizing enzymes, which are chiral molecules. Due to stereoselectivity, each drug enantiomer may be metabolized through different pathways at different rates by these chiral enzymes. As the metabolism of chiral mixtures may require different reactions for each of the individual isomers, this may raise the possibility of additional burden on the liver. Furthermore, one of the critical components of drug-induced liver injury has been reported as drug biotransformation . For instance, hepatotoxicity-related withdrawal of benoxaprofen, a racemic NSAID, was reported to be mediated by the formation of reactive acyl glucuronide metabolites. While our data was not empowered to infer a causal association between chiral mixtures and liver injury, the tendency of racemic/non-racemic mixture drugs towards hepatotoxicitydriven withdrawal seems to have biological plausibility. This warrants designation of further detailed studies with specific racemic drugs to investigate such causal relationship. Another common reason for withdrawal, cardiovascular ADRs were more frequently seen with chiral mixtures compared to that of achiral drugs, but similar to pure enantiomers. Though cardiovascular ADRs may also involve various mechanisms, a recent machine learning-based computational model study addressed biological binding and substructural chemical features of drugs in predicting cardiovascular ADRs. The relative complexity of chiral mixtures might have contributed to observed high share of cardiovascular ADRs as a reason for withdrawal in our study.

Since the 1950s, the production of synthetic drugs has become widespread and mostly racemates have been produced by this method. This might have paved the way for the withdrawal of chiral drugs introduced in 1960-2000 to be predominantly mixtures. On the contrary, the limited number of drugs withdrawn in the last 20 years are mostly pure enantiomers, which might be related to their increasing share among newly approved drugs . Additionally, drugs with different chiral characteristics in our study had similar durations till withdrawal, and this pattern was mostly maintained in sub-analyses based on the most common ADRs (only with one exception in dermatological reactions). These results suggest that both temporal parameters might be unrelated to chiral characteristics of the drugs.

The results of the study should be interpreted considering the limitations below. Chirality details of some drugs/medical products included in the publication, which was used to determine the drugs withdrawn between 1950 and 2014, could not be accessed. This resulted in a partial reduction in the number of products evaluated. In addition, ADRs that led to the withdrawal of the evaluated drugs were obtained directly from the sources used to identify the drugs, so these should not be considered as first-hand findings. Chiral characteristics-based design of the study should not suggest that examined drugs were withdrawn from the market solely due to their stereochemical properties. We aimed to point out the possible effect of chirality on the distribution of ADRs, which should be further addressed by detailed future studies.

In conclusion, this study revealed that the stereochemical properties of drugs are among the factors which should be considered for drug safety. The chiral distribution of the withdrawn drugs could differ according to the usage areas and the underlying reasons for withdrawal. While the share of racemic/non-racemic mixtures in drugs withdrawn from the market does not highly support the arguments in favour of them posing higher risk, tendency of these drugs towards hepatotoxicity and cardiovascular ADRs draws attention. The potential relationships between stereochemical properties of drugs and ADR mechanisms should be investigated with further experimental and epidemiological studies, which might eventually lead to improvements of safety standards in new drug development processes.

\section{Acknowledgements}

We thank to Dr. Igho Onakpoya and his co-workers for their tremendous efforts and earlier studies on withdrawn drugs that inspired us to investigate another aspect of the subject.

\section{Competing Interests}

The authors declare they have no competing or conflict of interest. 


\section{Funding}

This research did not receive any specific grant from funding agencies in the public, commercial, or not-forprofit sectors.

\section{Contributions}

$\mathrm{AB}, \mathrm{VA}$, and $\mathrm{AA}$ contributed to the study conceptualization and design. $\mathrm{AB}, \mathrm{CV}$, and VA collected the data. Analyses were performed by AB, VA, and CV. The first draft of the manuscript was written by VA, AB, and CV. AA contributed to the critical review and supervision of the study. All authors read and approved to the final version of the manuscript prior to submission.

\section{Data availability}

The data that support the findings of this study are available from the corresponding author upon reasonable request.

\section{References}

1. McConathy J, Owens MJ. Stereochemistry in Drug Action. Prim Care Companion J Clin Psychiatry. 2003;5(2):70-73. https://doi.org/10.4088/pcc.v05n0202.

2. Abram M, Jakubiec M, Kaminski K. Chirality as an important factor for the development of new antiepileptic drugs. Chem Med Chem. 2019;20(14):1744-1761. https://doi.org/ $10.1002 / \mathrm{cmdc} .201900367$.

3. Smith SW. Chiral toxicology: it's the same thing... only different. Toxicol Sci. 2009;110(1):4-30. https://doi.org/10.1093/toxsci/kfp097.

4. Wei X, Liu J, Xia GJ, Deng J, Sun P, Chruma JJ, Wu W, Yang C, Wang YG, Huang Z. Enantioselective photoinduced cyclodimerization of a prochiral anthracene derivative adsorbed on helical metal nanostructures. Nat Chem. 2020;12(6):551-559. https://doi.org/10.1038/s41557-020-0453-0.

5. Chmielewska A, Konieczna L, Bączek T. A novel two-step liquid-liquid extraction procedure combined with stationary phase immobilized human serum albumin for the chiral separation of cetirizine enantiomers along with $\mathrm{M}$ and $\mathrm{P}$ parabens. Molecules. 2016;21(12):1654. https://doi.org/10.3390/molecules21121654.

6. Tillement JP, Testa B, Brée F. Compared pharmacological characteristics in humans of racemic cetirizine and levocetirizine, two histamine H1-receptor antagonists. Biochem Pharmacol. 2003;66(7):1123-6. https://doi.org/10.1016/s0006-2952(03)00558-6.

7. Blaschke G, Kraft HP, Fickentscher K, Köhler F. Chromatographic separation of racemic thalidomide and teratogenic activity of its enantiomers. Arzneim.-Forsch. 1979;29(10):1640-1642.

8. Tokunaga E, Yamamoto $\mathrm{T}$, Ito $\mathrm{E}$, Shibata N. Understanding the thalidomide chirality in biological processes by the self-disproportionation of enantiomers. Sci Rep. 2018;8(1):17131. https://doi.org/10.1038/s41598-018-35457-6.

9. Agranat I, Caner H. Intellectual property and chirality of drugs. Drug Discovery Today. 1999;4(7):313321. https://doi.org/10.1016/s1359-6446(99)01363-x.

10. Gal J. New single-isomer compounds on the horizon. CNS Spectr. 2002;7(4 Suppl 1):45-54. https://doi.org/10.1017/S1092852900028601.

11. Tucker GT. Chiral switches. Lancet. 2000;355(9209):1085-7. https://doi.org/10.1016/S01406736(00)02047-X.

12. Onakpoya IJ, Heneghan CJ, Aronson JK. Post-marketing withdrawal of 462 medicinal products because of adverse drug reactions: a systematic review of the world literature. BMC Med. 2016;14:10. https://doi.org/10.1186/s12916-016-0553-2.

13. NIH National Center for Advancing Translational Sciences. Inxight: Drugs. https://drugs.ncats.ioAccessed 13 Jan 2022.

14. Nguyen LA, He H, Pham-Huy C. Chiral drugs: an overview. Int J Biomed Sci. 2006;2(2):85-100.

15. Katzung BG. Introduction: The Nature of Drugs \& Drug Development \& Regulation. In: Katzung BG, eds. Basic \& Clinical Pharmacology. 15th ed. McGraw-Hill Education: US; 2020.p: 1-20. 
16. Caner H, Groner E, Levy L, Agranat I. Trends in the development of chiral drugs. Drug Discov Today. 2004;9(3):105-10. https://doi.org/10.1016/s1359-6446(03)02904-0.

17. Lennard MS. Clinical pharmacology through the looking glass: reflections on the racemate vs enantiomer debate. Br J Clin Pharmacol. 1991;31(6):623-625. https://doi.org/10.1111/j.13652125.1991.tb05583.x.

18. Mwamwitwa KW, Kaibere RM, Fimbo AM, Sabitii W, Ntinginya NE, Mmbaga BT, Shewiyo DH, Shearer MC, Smith AD, Kaale EA. A Retrospective Cross-sectional Study to Determine Chirality Status of Registered Medicines in Tanzania. Sci Rep. 2020;10(17834). https://doi.org/10.1038/s41598020-74932-x.

19. Lin GQ, Zhang JG, Cheng JF. Overview of Chirality and Chiral Drugs. In: Chiral Drugs: Chemistry and Biological Action, 1st ed. Eds: Lin GQ, Zhang JG, Cheng JF. John Wiley \& Sons, Inc. 2011, p. 3-28. https://doi.org/10.1002/9781118075647.ch1.

20. Agranat I, Caner H, Caldwell J. Putting chirality to work: the strategy of chiral switches. Nat Rev Drug Discov. 2002;1(10):753-768. https://doi.org/10.1038/nrd915.

21. WHO Collaborating Centre for Drug Statistics Methodology, ATC classification index with DDDs, 2021. Oslo, Norway 2020. https://www.whocc.no/atc_ddd_index/Accessed 29 Dec 2021.

22. Fung M, Thornton A, Mybeck K, Wu JH, Hornbuckle K, Muniz E. Evaluation of the Characteristics of Safety Withdrawal of Prescription Drugs from Worldwide Pharmaceutical Markets-1960 to 1999. Drug Inf J. 2001;35(1):293-317. https://doi.org/10.1177/009286150103500134.

23. Inotai A, Hankó B, Mészáros Á. Trends in the non-steroidal anti-inflammatory drug market in six Central-Eastern European countries based on retail information. Pharmacoepidemiol Drug Saf. 2010;19(2):183-90. https://doi.org/10.1002/pds.1893.

24. Ranade VV, Somberg JC. Chiral cardiovascular drugs: an overview. Am J Ther. 2005;12(5):439-59. https://doi.org/10.1097/01.mjt.0000167429.37357.0c.

25. Alomar MJ. Factors affecting the development of adverse drug reactions (Review article). Saudi Pharm J. 2014;22(2):83-94. https://doi.org/10.1016/j.jsps.2013.02.003.

26. Gross AS, Somogyi A, Eichelbaum M. Stereoselective Drug Metabolism and Drug Interactions. In: Eichelbaum M, Somogyi A eds, Stereochemical Aspects of Drug Action and Disposition. Handbook of Experimental Pharmacology, vol 153. Berlin: Springer; 2003, p:313-339. https://doi.org/10.1007/9783-642-55842-9_13.

27. Faber K, Griengl H. Chirality in Organic Synthesis - The Use of Biocatalysts. In: Janoschek R., eds. Chirality. Berlin: Springer; 1991, p:103-140.

28. European Association for the Study of the Liver. EASL clinical practice guidelines: Drug-induced liver injury. J Hepatol 2019;70:1222-61. https://doi.org/10.1016/j.jhep.2019.02.014.

29. Walgren JL, Mitchell MD, Thompson DC. Role of metabolism in drug-induced idiosyncratic hepatotoxicity. Crit Rev Toxicol. 2005;35(4):325-61. https://doi.org/10.1080/10408440590935620.

30. Jamal S, Ali W, Nagpal P, Grover S, Grover A. Computational models for the prediction of adverse cardiovascular drug reactions. J Transl Med. 2019;17(1):171. https://doi.org/10.1186/s12967-019-1918z.

31. Blaser HU. Chirality and its implications for the pharmaceutical industry. Rend. Fis. Acc. Lincei 2013;24:213-216. https://doi.org/10.1007/s12210-012-0220-2.

Tables

Table 1. Comparison of chiral distribution of the withdrawn drugs at ATC-1 level.

\begin{tabular}{|c|c|c|c|c|c|c|c|}
\hline ATC-1 Code & $\begin{array}{l}\text { Total } \\
\mathbf{n}\end{array}$ & $\begin{array}{l}\text { Total } \\
\%\end{array}$ & $\begin{array}{l}\text { Achiral drugs } \\
\mathbf{n}\end{array}$ & $\begin{array}{l}\text { Achiral drugs } \\
\%\end{array}$ & $\begin{array}{l}\text { Chiral mixtures } \\
\text { n }\end{array}$ & $\begin{array}{l}\text { Chiral mixtures } \\
\%\end{array}$ & $\begin{array}{l}\text { Pure enas } \\
\mathbf{n}\end{array}$ \\
\hline A & 62 & 15.7 & 28 & 13.5 & 17 & 15.6 & 17 \\
\hline B & 3 & 0.8 & 1 & 0.5 & - & - & 2 \\
\hline C & 45 & 11.4 & 16 & 7.7 & 19 & 17.5 & 10 \\
\hline $\mathrm{D}$ & 15 & 3.8 & 11 & 5.3 & - & - & 4 \\
\hline G & 16 & 4.0 & 7 & 3.4 & 2 & 1.8 & 7 \\
\hline
\end{tabular}




\begin{tabular}{llllllll}
$\mathrm{J}$ & 30 & 7.6 & 16 & 7.7 & 6 & 5.5 & 8 \\
$\mathrm{~L}$ & 8 & 2.0 & 5 & 2.4 & 3 & 2.8 & - \\
$\mathrm{M}$ & 41 & 10.4 & 26 & 12.6 & 13 & 30.3 & 2 \\
$\mathrm{~N}$ & 104 & 26.3 & 56 & 27.1 & 33 & 1.9 & 3 \\
$\mathrm{P}$ & 18 & 4.6 & 13 & 6.3 & 2 & 9.2 & 4 \\
$\mathrm{R}$ & 26 & 6.6 & 12 & 5.8 & 10 & 0.9 & 3 \\
$\mathrm{~S}$ & 6 & 1.5 & 2 & 1.0 & 1 & 0.9 & 3 \\
$\mathrm{~V}$ & 9 & 2.3 & 5 & 2.4 & 1 & 1.9 & 1 \\
Miscellaneous & 12 & 3.0 & 9 & 4.3 & 2 & 100.0 & 79 \\
Total & 395 & 100.0 & 207 & 100.0 & 109 & & \\
\hline
\end{tabular}

${ }^{*} \mathrm{p}=0.01$ for achiral vs. racemic/non-racemic mixture drugs. ${ }^{+} \mathrm{p}=0.04$ for racemic/non-racemic mixture vs. pure enantiomers. ${ }^{++} \mathrm{p}=0.01$ and $\mathrm{p}=0.03$ for pure enantiomers vs. achiral and racemic/non-racemic mixture drugs, respectively. A, Alimentary tract and metabolism; B, Blood and blood forming organs; $C$, Cardiovascular system; D, Dermatological; G, Genitourinary system and sex hormones; J, Anti-infectives for systemic use; L, Antineoplastic and immunomodulating agents; M, Musculoskeletal system; N, Nervous system; P, Antiparasitic products, insecticides and repellents; $R$, Respiratory system; S, Sensory organs; V, Various. Miscellaneous denotes drugs with multiple or undefined ATC codes.

Table 2. Comparison of chirality status of drugs leading to most common ADRs ( $>30)$.

\begin{tabular}{lllllll}
\hline Adverse drug reaction & $\begin{array}{l}\text { Achiral } \\
\mathbf{n}\end{array}$ & $\begin{array}{l}\text { Achiral } \\
\mathbf{\%}\end{array}$ & $\begin{array}{l}\text { Chiral mixture } \\
\mathbf{n}\end{array}$ & $\begin{array}{l}\text { Chiral mixture } \\
\mathbf{\%}\end{array}$ & $\begin{array}{l}\text { Pure enantiomer } \\
\mathbf{n}\end{array}$ & $\begin{array}{l}\text { Pure enan } \\
\text { \% }\end{array}$ \\
Hepatotoxicity & 52 & 17.0 & 21 & 12.7 & 6 & 5.4 \\
No hepatotoxicity & 254 & 83.0 & 144 & 87.3 & 106 & 94.6 \\
Cardiovascular & 23 & 7.5 & 24 & 14.5 & 13 & 11.6 \\
Non-cardiovascular & 283 & 92.5 & 141 & 85.5 & 99 & 88.4 \\
Drug abuse & 16 & 5.2 & 22 & 13.3 & 13 & 88.4 \\
No drug abuse & 290 & 94.8 & 143 & 86.7 & 99 & 10.7 \\
Neurological & 27 & 8.8 & 11 & 6.7 & 12 & 89.3 \\
Non-neurological & 279 & 91.2 & 154 & 93.3 & 100 & 9.8 \\
Haematological & 30 & 9.8 & 8 & 4.8 & 11 & 90.2 \\
Non-haematological & 276 & 90.2 & 157 & 95.2 & 101 & 8.0 \\
Carcinogenicity & 28 & 9.2 & 7 & 4.2 & 9 & 92.0 \\
No carcinogenicity & 278 & 90.8 & 158 & 95.8 & 103 & 98.2 \\
Dermatological & 27 & 8.8 & 9 & 5.5 & 2 & 6.2 \\
Non-dermatological & 279 & 91.2 & 156 & 94.5 & 110 & 93.8 \\
Immunological & 15 & 4.9 & 8 & 4.8 & 7 & 100.0 \\
Non-immunological & 291 & 95.1 & 157 & 95.2 & 105 & \\
Total & 306 & 100.0 & 165 & 100.0 & 112 & \\
\hline
\end{tabular}

${ }^{*} \mathrm{p}<0.01$ for pure enantiomer vs. achiral and $\mathrm{p}=0.04$ for pure enantiomer vs. chiral mixtures; ${ }^{+} \mathrm{p}=0.02$ for achiral vs. chiral mixtures; ${ }^{++} \mathrm{p}<0.01$ for achiral vs. chiral mixtures and $\mathrm{p}=0.03$ for achiral vs. pure enantiomers $;{ }^{\delta} \mathrm{p}<0.01$ for pure enantiomer vs. achiral drugs.

Table 3. Chiral distribution of the drugs by the time after launch.

\begin{tabular}{lllllll}
\hline Year of launch & Achiral drugs & Achiral drugs & Chiral mixtures & Chiral mixtures & Pure enantiomers & Pu \\
& $\mathbf{n}$ & $\%$ & $\mathbf{n}$ & $\mathrm{n}$ & $\%$
\end{tabular}




\begin{tabular}{lllllll} 
Before 1920 & 17 & 70.8 & 1 & 4.2 & 6 & 25. \\
$1920-1940$ & 15 & 53.6 & 7 & 25.0 & 6 & 21. \\
$1940-1960$ & 48 & 51.6 & 24 & 25.8 & 21 & 22. \\
$1960-1980$ & 74 & 51.0 & 46 & 31.7 & 15 & 17. \\
$1980-2000$ & 42 & 48.3 & 30 & 34.5 & 6 & 33. \\
After 2000 & 11 & 61.1 & 1 & 5.6 & 79 & 20. \\
Total & 207 & 52.4 & 109 & 27.6 & & \\
\hline
\end{tabular}

${ }^{*}$ The column indicates relative share of the time period for all withdrawn drugs.

\section{Table 4. Comparison of time to withdrawal of chiral groups for the most common}

\section{ADRs.}

\begin{tabular}{|c|c|c|c|c|c|}
\hline Adverse drug reactions $(\mathrm{n}[?] 30)$ & $\begin{array}{l}\text { Achiral } \\
\text { Mean }\end{array}$ & $\begin{array}{l}\text { Achiral } \\
\pm \mathrm{SD}\end{array}$ & $\begin{array}{l}\text { Chiral mixture } \\
\text { Mean }\end{array}$ & $\begin{array}{l}\text { Chiral mixture } \\
\pm \mathrm{SD}\end{array}$ & $\begin{array}{l}\text { Pure enantiomer } \\
\text { Mean }\end{array}$ \\
\hline Hepatotoxicity $(\mathrm{n}=79)$, yrs. & 19.1 & \pm 25.8 & 13.8 & \pm 12.7 & 12.3 \\
\hline Cardiovascular $(\mathrm{n}=60)$, yrs. & 17.7 & \pm 27.4 & 23.2 & \pm 14.4 & 35.9 \\
\hline Drug abuse $(n=51)$, yrs. & 31.9 & \pm 33.0 & 31.5 & \pm 16.7 & 45.1 \\
\hline Neurological $(\mathrm{n}=50)$, yrs. & 28.9 & \pm 27.6 & 18.8 & \pm 18.6 & 53.3 \\
\hline Haematological $(n=49)$, yrs. & 38.4 & \pm 31.5 & 16.1 & \pm 13.3 & 20.0 \\
\hline Carcinogenicity $(\mathrm{n}=44)$, yrs. & 39.2 & \pm 40.5 & 17.9 & \pm 21.4 & 11.7 \\
\hline Dermatological $(\mathrm{n}=38)$, yrs. & 28.4 & \pm 20.4 & 9.3 & \pm 10.9 & 18.0 \\
\hline Immunological $(n=30)$, yrs. & 38.1 & \pm 29.4 & 15.1 & \pm 13.9 & 21.3 \\
\hline
\end{tabular}

${ }^{*} \mathrm{p}>0.05$ for each of the multiple comparisons between pairs. ${ }^{+} \mathrm{p}=0.01$ for achiral drugs vs. chiral mixtures.

Figure Legends

Figure 1. Chiral distribution of the withdrawn drugs at the ATC-1 level. ${ }^{*} p=0.02$ for achiral vs. chiral drugs. [A, Alimentary tract and metabolism ( $n=62) ; B$, Blood and blood forming organs ( $n=3)$; $C$, Cardiovascular system ( $n=45) ; D$, Dermatological $(n=15) ; G$, Genito urinary system and sex hormones ( $n=13) ; J$, Anti-infectives for systemic use (n=30); L, Antineoplastic and immunomodulating agents $(n=8) ; M$, Musculoskeletal system ( $n=41) ; N$, Nervous system ( $n=104) ; P$, Antiparasitic products, insecticides and repellents $(n=18) ; R$, Respiratory system ( $n=26) ; S$, Sensory organs $(n=6) ; V$, Various ( $n=9) ;$ Others denotes drugs with multiple or undefined ATC codes ( $n=12)]$ 


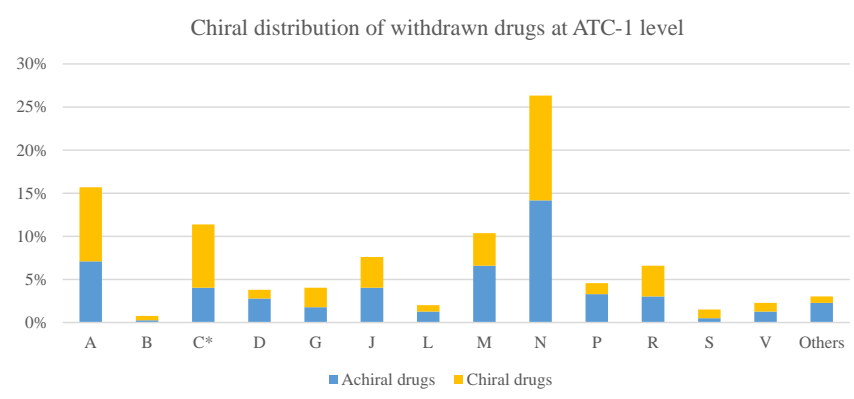

